\title{
Social Assistance and Minimum Income Levels and Replacement Rates Dataset
}

\author{
Assembled by Jinxian Wang and Olaf van Vliet ${ }^{*}$
}

Leiden University

December 2016

\begin{abstract}
The Social Assistance and Minimum Income Levels and Replacement Rates Dataset provides two new indicators for comparisons of social assistance and minimum income benefits across countries and over time, namely real net minimum income benefit levels and net minimum income benefit replacement rates. The dataset contains information for 33 countries for the period 1990 - 2009. For information on social assistance and minimum income benefits, the dataset draws upon information from Nelson's (2013) dataset. The minimum income replacement rates are comparable to unemployment replacement rates.
\end{abstract}

\section{Introduction}

Until recently, comparative studies on social policy and labor market institutions have paid little attention to social assistance and minimum income benefits compared to other welfare state programs such as labor market policies and pension schemes. One explanation for this lack of attention might be that data on other welfare state programs, most notably social expenditure data provided by the OECD, were relatively easily available, whereas data on social assistance and minimum income benefits were not. This came to an end in 2006, when Nelson published the first version of the Social Assistance and Minimum Income Protection Dataset. More recently, Van Mechelen et al. (2011) published the CSB-Minimum Income Protection Indicators Dataset.

\footnotetext{
*E-mail: j.wang@law.leidenuniv.nl and o.p.van.vliet@law.leidenuniv.nl. Department of Economics, Leiden University. The dataset is available from the following website:

www.universiteitleiden.nl/en/law/institute-for-tax-law-and-economics/economics/data-sets
} 
To compare social policies across countries and over time, many empirical studies have relied on social expenditure data, but these are not available for minimum income benefits. Furthermore, comparative studies of welfare state programs have increasingly turned to the use of social right indicators, such as income replacement rates. These studies are mainly focused on international comparative analyses of unemployment benefits, sick pay benefits and pension benefits (e.g. Allan and Scruggs, 2004; Hicks and Freeman, 2009; Korpi and Palme, 2003; Scruggs et al., 2014 and Van Vliet and Caminada, 2012). Much less attention has been paid to the international comparative analysis of minimum income benefits.

The aim of the Social Assistance and Minimum Income Levels and Replacement Rates Dataset, assembled by Wang and Van Vliet, is to provide an essential dataset for all kind of cross-national analyses related to minimum income benefit programs. We introduce two types of quantitative indicators of minimum income benefits, namely the real net minimum income benefit levels and the net minimum income replacement rates. The benefit level is a relatively straightforward measure of (one dimension of) the generosity of minimum income benefits (Olaskoaga et al., 2013). The minimum income replacement rate, on the other hand, gives an impression of the benefit generosity relative to labor income. These indicators are based on data from the Social Assistance and Minimum Income Protection Dataset (Nelson, 2013) and the Unemployment Replacement Rates Dataset among 34 Welfare States, 1971-2009: An Update, Extension and Modification of the Scruggs' Welfare State Entitlement Data Set (Van Vliet and Caminada, 2012).

The Social Assistance and Minimum Income Levels and Replacement Rates Dataset allows researchers and public policy analysts to compare the generosity of minimum income benefits across 33 countries between 1990-2009. The data can be used to address questions of how the generosity of welfare states varies across countries and over time. Subsequently, researchers can use the data to explore the sources of this variation (Wang et al., 2015) Furthermore, the dataset can be used by scholars to examine the effects of benefit programs on labor market outcomes (Been and Van Vliet, 2017).

\section{Origin of the idea}

Social Assistance and Minimum Income Levels and Replacement Rates Dataset, assembled by Wang and Van Vliet (Version December 2016), builds upon two other datasets. Firstly, data on social assistance and minimum income benefits is provided by Nelson's (2013) Social Assistance and Minimum Income Protection Dataset. Secondly, it elaborates on the concept of net income 
replacement rates as used in the work on unemployment replacement rates, developed by Scruggs et al. (2014) and Van Vliet and Caminada (2012).

\section{Citation}

A more detailed description of the data and method is available in the following article:

Wang, J., and O. van Vliet (2016) Social assistance and minimum income benefits: Benefit levels, replacement rates and policies across 26 OECD countries, 1990-2009. European Journal of Social Security 18(4): 333-555.

Please cite this article when referring to the data.

\section{Definition: real net minimum income benefit levels}

To construct the indicator of real net minimum income benefits, our dataset relies on data from the Social Assistance and Minimum Income Protection Dataset (Nelson, 2013). Nelson's (2013) dataset contains annual data on benefit levels for a fairly large number of industrialized countries. The institutional information in the dataset is based on national sources and legislation. The dataset contains disaggregated data on several income components. Following Nelson (2013), we define net minimum income benefits as the net income from a benefit package consisting of basic social assistance, child supplements, refundable tax credits and other benefits. One-time social assistance payments to cover unexpected and urgent needs or regular supplements to cover exceptional needs are not included in this benefit package.

Furthermore, housing benefits are not included in the calculation of our main indicators of net minimum income benefits. In many countries, housing benefits depend on actual housing costs. As the actual housing costs vary strongly across regions within countries, the inclusion of housing benefits in the calculated benefit package requires demanding assumptions on the housing costs. Consequently, the assumptions regarding the housing costs strongly determine the calculated housing benefits and so the calculated net minimum income benefit levels (Eardly et al., 1996; Kuivalainen, 2003; Van Mechelen et al, 2011). The consequence of the decision not to include housing benefits is that our indicators underestimate the minimum income, but the advantage is that our indicators give a clearer indication of the development of social assistance benefits. Moreover, not including the housing benefits in the minimum income benefit package has the advantage that the benefit package is comparable to the benefit package used in studies on unemployment benefits. 
Therefore, our minimum income replacement rates are comparable to unemployment replacement rates (Scruggs et al., 2014; Van Vliet and Caminada, 2012).

Nevertheless, the dataset also includes a measure of minimum income benefit levels including housing benefits. These benefit levels are higher than the benefit levels without housing benefits. With regard to the developments between 1990 and 2009, these are comparable for the measures with and without housing benefits for all countries but Sweden.

In order to compare benefit levels across countries and over time, we transfer all benefit levels into U.S. dollars, adjusted for purchasing power (PPP) and inflation (CPI=100). Data on PPP are taken from Penn World Table (Heston et al., 2012) and for the CPI we rely on data from the World Bank (2012). An additional step is required for the Eurozone countries, as the PPPs in the year before the introduction of the euro are also expressed in euros, whereas the benefit levels are expressed in the national currencies. To convert the national currencies into euros, our dataset relies on exchange rate data from the European Commission (2014).

For the Eurozone countries, the real minimum income benefit levels are calculated as follows:

Real minimum income benefit $=(($ minimum income benefit/exchange rate from euro to national currency)/PPP)*(100/CPI)

For the non-Eurozone countries, the real minimum income benefit levels are calculated as follows:

Real minimum income benefit $=($ minimum income benefit $/ P P P) *(100 / C P I)$

The dataset contains data for three different household types: single person households, lone parent households with two children, and two parent households with two children.

\section{Definition: net minimum income benefit replacement rates}

The net minimum income replacement rate is defined as the ratio of the net minimum income benefits to the net average production worker wage. For the replacement rate, we neither adjust the nominator nor the denominator for exchange rates, purchasing power, or inflation, as these adjustments would be cancelled out in the ratio. The net average production worker wage refers to the in-work wage after deducting taxes. Data on minimum income benefits are taken from the Social Assistance and Minimum Income Protection Dataset (Nelson, 2013). For the average production worker wage, we use data from the OECD and Unemployment Replacement Rates Dataset among 34 
Welfare States, 1971-2009: An Update, Extension and Modification of the Scruggs' Welfare State Entitlement Data Set (Van Vliet and Caminada, 2012).

It should be noted that in most cases, minimum income 'replacement rates' do not indicate the fraction of the income from work that is actually 'replaced' by income transfer programs, as is for instance the case for unemployment replacement benefits. The reason for this difference is that unemployed workers often receive unemployment benefits first before they are entitled to social assistance benefits. Hence, minimum income replacement rates and unemployment replacement rates are the same type of ratios as they share the same denominator, but the meaning of the term 'replacement rate' is slightly different.

\section{Summary statistics}

The tables below provide a summary of the data. Tables $1-3$ present the real net minimum income benefit levels for a single person household without children, a lone parent household with two children and a two parent household with two children respectively. Table 4 presents a summary measure of the real net minimum income benefit levels (the mean of the three household types).

Tables $5-7$ present the net minimum income replacement rates for a single person household without children, a lone parent household with two children and a two parent household with two children respectively. Table 8 presents a summary measure of the net minimum income replacement rates (the mean of the three household types). 
Table 1. Real net minimum income benefit levels for single person household without children

\begin{tabular}{|c|c|c|c|c|c|c|c|c|c|c|c|c|c|c|c|c|c|c|c|c|}
\hline & 1990 & 1991 & 1992 & 1993 & 1994 & 1995 & 1996 & 1997 & 1998 & 1999 & 2000 & 2001 & 2002 & 2003 & 2004 & 2005 & 2006 & 2007 & 2008 & 2009 \\
\hline Australia & 7,101 & 7,462 & 7,698 & 7,735 & 7,712 & 7,711 & 7,910 & 8,137 & 8,037 & 7,968 & 7,528 & 7,744 & 7,755 & 7,800 & 7,926 & 7,941 & 8,093 & 8,124 & 8,030 & 8,179 \\
\hline Austria & 5,095 & 4,933 & 5,460 & 5,661 & 5,603 & 5,786 & 5,703 & 5,533 & 5,525 & 5,698 & 5,701 & 5,703 & 5,832 & 5,754 & 5,869 & 5,859 & 6,059 & 6,103 & 6,155 & 6,255 \\
\hline Belgium & 7,742 & 7,998 & 7,993 & 8,222 & 8,290 & 8,671 & 8,600 & 8,205 & 8,177 & 8,228 & 8,168 & 8,130 & 8,485 & 8,506 & 8,682 & 8,545 & 8,407 & 8,778 & 8,916 & 9,391 \\
\hline Bulgaria & & . & . & & & . & & . & & & . & & . & . & & . & & 1,231 & 1,039 & 1,196 \\
\hline Canada & 8,099 & 8,496 & 8,693 & 8,667 & 8,824 & 8,271 & 6,826 & 6,741 & 6,608 & 6,550 & 6,379 & 6,226 & 6,092 & 5,982 & 6,042 & 6,037 & 5,974 & 6,080 & 6,238 & 6,099 \\
\hline Cyprus & . & . & . & & . & . & & . & 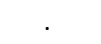 & . & . & & . & . & 5,786 & 5,860 & 6,007 & 5,799 & 6,237 & 7,021 \\
\hline Czech Republic & . & . & . & 4,268 & 3,911 & 3,734 & 3,494 & 3,445 & 3,284 & 3,173 & 3,006 & 3,398 & 3,367 & 3,408 & 3,279 & 3,452 & 3,493 & 1,980 & 1,873 & 1,855 \\
\hline Denmark & 5,059 & 5,064 & 5,227 & 5,297 & 7,269 & 8,700 & 8,637 & 8,545 & 8,623 & 8,674 & 8,712 & 8,789 & 8,845 & 8,999 & 9,257 & 9,460 & 9,618 & 9,748 & 9,440 & 9,762 \\
\hline Estonia & . & . & . & . & . & 1,354 & 1,109 & 1,118 & 1,047 & 982 & 919 & 846 & 806 & 797 & 769 & 1,095 & 1,013 & 1,078 & 1,059 & 1,085 \\
\hline Finland & 6,149 & 5,673 & 4,913 & 4,243 & 4,631 & 5,126 & 4,990 & 4,888 & 4,689 & 4,724 & 4,576 & 4,588 & 4,624 & 4,687 & 4,805 & 4,884 & 4,915 & 4,878 & 4,753 & 4,918 \\
\hline France & 4,481 & 4,435 & 4,491 & 4,684 & 4,788 & 4,855 & 4,966 & 4,929 & 4,962 & 5,173 & 5,188 & 5,276 & 5,222 & 5,250 & 5,216 & 5,339 & 5,346 & 5,425 & 5,423 & 5,565 \\
\hline Germany & . & 4,424 & 4,428 & 4,494 & 4,462 & 4,521 & 4,456 & 4,309 & 4,325 & 4,443 & 4,532 & 4,551 & 4,575 & 4,626 & 4,655 & 5,488 & 5,533 & 5,542 & 5,499 & 5,616 \\
\hline Hungary & . & . & 6,996 & 7,877 & 7,831 & 4,992 & 3,473 & 2,591 & 2,087 & 1,846 & 1,690 & 1,562 & 1,547 & 1,561 & 1,615 & 1,562 & 1,772 & 2,288 & 1,849 & 1,743 \\
\hline Ireland & 6,983 & 7,077 & 7,001 & 6,167 & 6,371 & 6,655 & 6,861 & 7,430 & 7,166 & 6,937 & 6,702 & 6,761 & 6,970 & 6,932 & 7,252 & 7,898 & 8,464 & 9,137 & 9,645 & 10,771 \\
\hline Italy & 10,307 & 9,867 & 9,158 & 7,484 & 6,841 & 5,863 & 5,977 & 5,873 & 5,725 & 6,811 & 6,850 & 7,028 & 7,110 & 7,265 & 7,457 & 7,764 & 8,079 & 8,325 & 8,555 & 8,909 \\
\hline Japan & 5,344 & 5,394 & 5,547 & 5,674 & 5,739 & 6,012 & 6,122 & 5,998 & 6,179 & 6,367 & 6,661 & 6,936 & 7,202 & 7,394 & 7,644 & 8,011 & 8,260 & 8,518 & 8,636 & 8,968 \\
\hline Latvia & . & . & . & . & . & . & . & . & . & . & . & . & . & . & 744 & 764 & 772 & 708 & 557 & 754 \\
\hline Lithuania & . & . & . & . & . & . & . & . & . & . & . & . & . & . & 991 & 947 & 996 & 1,187 & 1,429 & 1,740 \\
\hline Luxembourg & 8,617 & 9,189 & 9,555 & 11,413 & 11,550 & 12,343 & 12,078 & 12,082 & 11,941 & 12,059 & 11,850 & 12,198 & 12,249 & 12,733 & 12,911 & 13,320 & 13,450 & 13,891 & 13,932 & 16,701 \\
\hline Malta & . & . & . & . & . & . & . & . & . & . & . & & . & . & 6,582 & 7,591 & 7,853 & 7,017 & 6,920 & 6,879 \\
\hline Nether & 10,263 & 10,385 & 10,534 & 10,916 & 10,797 & 11,673 & 11,262 & 10,704 & 10,405 & 10,501 & 10,425 & 10,566 & 10,386 & 10,530 & 10,806 & 10,739 & 11,190 & 11,540 & 11,745 & 11,719 \\
\hline New Z & 5,731 & 5,155 & 5,189 & 5,219 & 5,214 & 5,162 & 5,190 & 5,262 & 5,209 & 5,248 & 5,429 & 5,374 & 5,372 & 5,546 & 5,543 & 6,005 & 6,034 & 6,091 & 6,088 & 6,078 \\
\hline Norway & 7,715 & 7,634 & 7,654 & 7,530 & 8,472 & 9,215 & 10,017 & 11,636 & 9,457 & 10,717 & 10,403 & 10,467 & 10,653 & 10,703 & 10,718 & 10,706 & 10,658 & 10,642 & 10,265 & 9,936 \\
\hline Poland & . & . & . & . & . & 6,851 & 4,860 & 3,742 & 3,583 & 3,175 & 2,828 & 2,973 & 2,978 & 3,016 & 2,897 & 2,881 & 2,895 & 2,910 & 2,789 & 2,658 \\
\hline Portugal & . & . & . & . & . & . & 2,397 & 2,358 & 2,344 & 2,426 & 2,464 & 2,444 & 2,458 & 2,442 & 2,553 & 2,699 & 2,748 & 2,794 & 2,798 & 2,944 \\
\hline Romania & . & . & . & & . & . & & . & & & . & & . & & & . & & 603 & 530 & 522 \\
\hline Slovakia & . & . & . & 4,095 & 2,976 & 2,715 & 2,852 & 3,066 & 2,983 & 2,524 & 2,334 & 2,179 & 2,281 & 1,625 & 1,555 & 1,597 & 1,676 & 1,986 & 2,074 & 2,123 \\
\hline Slovenia & . & . & . & $\cdot$ & . & . & $\cdot$ & 4,549 & 3,908 & 3,429 & 3,106 & 2,608 & 4,334 & 3,951 & 3,717 & 3,796 & 3,789 & 3,810 & 3,826 & 3,820 \\
\hline Spain & 11,390 & 10,450 & 9,240 & 7,595 & 6,590 & 6,271 & 6,046 & 5,658 & 5,431 & 5,334 & 5,304 & 5,584 & 5,451 & 5,321 & 5,195 & 5,178 & 5,107 & 5,182 & 5,184 & 5,487 \\
\hline Sweden & 5,461 & 5,181 & 5,361 & 5,079 & 5,052 & 4,948 & 4,994 & 4,323 & 4,392 & 4,304 & 4,415 & 4,289 & 4,358 & 4,447 & 4,669 & 4,731 & 4,809 & 4,828 & 4,797 & 4,783 \\
\hline Switzerlar & 6,748 & 6,773 & 6,817 & 6,669 & 6,749 & 6,806 & 6,804 & 6,786 & 7,087 & 7,627 & 7,589 & 7,674 & 7,839 & 7,923 & 7,997 & 6,898 & 6,867 & 6,858 & 6,737 & 6,982 \\
\hline United Kingdom & 4,803 & 4,676 & 4,807 & 4,793 & 4,888 & 4,779 & 4,733 & 4,928 & 4,893 & 4,933 & 5,055 & 5,083 & 5,108 & 5,106 & 5,215 & 5,169 & 5,166 & 5,287 & 5,224 & 5,334 \\
\hline United States & 1,776 & 1,807 & 1,854 & 1,801 & 1,771 & 1,769 & 1,778 & 1,752 & 1,754 & 440 & 432 & 430 & 440 & 443 & 437 & 447 & 442 & 438 & 441 & 451 \\
\hline
\end{tabular}


Table 2. Real net minimum income benefit levels for lone parent household with two children

\begin{tabular}{|c|c|c|c|c|c|c|c|c|c|c|c|c|c|c|c|c|c|c|c|c|}
\hline & 1990 & 1991 & 992 & 1993 & 994 & 1995 & 1996 & 1997 & 1998 & 1999 & 2000 & 2001 & 2002 & 2003 & 2004 & 2005 & 2006 & 2007 & 2008 & 2009 \\
\hline Australia & 12,477 & 12,835 & 243 & 643 & 831 & 650 & 018 & 15,296 & 15,106 & 15,087 & 14,323 & 16,173 & 16,135 & 16,277 & 17,403 & 17,392 & 17,593 & 17,684 & 17,935 & 18,265 \\
\hline Austria & 993 & ,613 & 200 & 734 & 648 & 957 & 2,456 & 2,084 & 2,035 & 2,634 & 12,767 & 2,755 & 2,942 & 2,984 & 3,136 & 3,078 & 3,363 & 3,398 & 13,722 & 13,802 \\
\hline Belgium & 1,808 & 4,688 & 4,988 & 5,418 & 5,495 & 6,208 & 16,076 & 15,424 & 15,179 & 15,332 & 15,133 & 15,063 & 15,587 & 15,548 & 15,791 & 5,620 & 15,643 & 16,337 & 16,805 & 18,049 \\
\hline ulga & & & & & . & & & & & & & & & & . & & & 3,720 & 3,348 & 3,852 \\
\hline & 9,136 & 9,888 & 20,144 & 0,082 & 0,431 & 9,248 & 16,265 & 16,025 & 15,899 & 15,945 & 15,814 & 15,704 & 15,537 & 15,376 & 15,630 & 15,879 & 17,925 & 17,879 & 17,225 & 17,016 \\
\hline Cyprus & . & . & . & 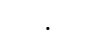 & . & . & . & & & . & . & . & & . & 10,860 & 10,993 & 11,251 & 10,899 & 11,551 & 12,945 \\
\hline Czech Republic & . & . & . & 10,125 & 9,342 & 9,028 & 8,248 & 8,217 & 7,680 & 7,419 & 7,028 & 7,725 & 7,653 & 7,746 & 7,454 & 7,778 & 7,840 & 4,901 & 4,636 & 4,592 \\
\hline Denmark & 11,802 & 12,015 & 12,579 & 12,741 & 14,537 & 14,666 & 14,688 & 14,657 & 14,821 & 14,886 & 14,694 & 14,771 & 14,873 & 15,131 & 15,544 & 16,197 & 16,123 & 16,326 & 15,808 & 16,245 \\
\hline & . & . & . & . & . & 3,521 & 2,883 & 2,908 & 2,722 & 2,552 & 2,390 & 2,201 & 2,096 & 2,071 & 2,000 & 2,847 & 2,633 & 2,803 & 2,754 & 3,038 \\
\hline Finland & 14,512 & 13,389 & 11,594 & 10,015 & 10,931 & 11,689 & 11,379 & 11,144 & 10,691 & 10,770 & 10,433 & 10,461 & 10,544 & 10,686 & 10,956 & 11,136 & 11,206 & 11,122 & 10,836 & 11,214 \\
\hline France & ,767 & 7,686 & 784 & 118 & 298 & 415 & 8,607 & 3,536 & 8,600 & 9,053 & 9,339 & 9,292 & 9,399 & 9,448 & 9,389 & 9,612 & 9,622 & 763 & 762 & 10,018 \\
\hline Germany & . & 10,498 & 10,860 & 1,492 & 1,428 & 11,593 & 11,436 & 11,048 & 11,106 & 11,267 & 11,490 & 11,532 & 11,449 & 11,575 & 11,647 & 13,492 & 13,603 & 13,625 & 13,518 & 13,813 \\
\hline ing & . & . & 17,402 & 16,965 & 14,357 & 9,061 & 6,489 & 4,968 & 4,096 & 3,702 & 3,240 & 2,878 & 2,735 & 2,891 & 2,904 & 2,862 & 4,004 & 6,365 & 6,183 & 5,829 \\
\hline Ireland & 11,355 & 11,390 & 11,228 & 10,146 & 10,359 & 10,940 & 11,138 & 11,997 & 11,327 & 11,013 & 10,597 & 10,463 & 10,833 & 11,806 & 12,179 & 12,909 & 13,714 & 14,932 & ,722 & 17,562 \\
\hline Italy & 213 & 21,252 & 19,723 & ,119 & 1,754 & 2,633 & 12,878 & 654 & 12,334 & 14,673 & 14,740 & 15,152 & 15,3 & 15,676 & 16,096 & 766 & & & & 19,269 \\
\hline Jap & 14,199 & 14,140 & 14,409 & 14,670 & 4,766 & 15,694 & 15,983 & 15,942 & 15,985 & 16,515 & 17,264 & 17,976 & 18,666 & 19,158 & 20,272 & 21,210 & 21,853 & 22,535 & 22,846 & 23,726 \\
\hline & . & . & . & . & . & . & . & . & . & . & . & . & & . & 2,231 & 2,291 & 2,317 & 2,125 & 1,670 & 2,263 \\
\hline & . & . & . & . & . & . & . & . & & . & . & . & & . & 2,973 & 2,840 & 2,988 & 3,562 & 3,766 & 5,219 \\
\hline Luxemb & 14,291 & 15,067 & 15,497 & 19,624 & 19,860 & 21,068 & 20,671 & 20,479 & 19,639 & 20,539 & 20,162 & 20,594 & 21,386 & 22,003 & 22,310 & 22,882 & 23,105 & 23,564 & 23,470 & 31,447 \\
\hline Malta & . & . & . & . & . & . & . & & & 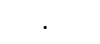 & . & . & & . & 10,753 & 11,328 & & 10,962 & & 11,637 \\
\hline & 6,220 & 16,406 & 6,637 & & & 18,189 & 17,599 & 16,774 & 16,390 & 16,515 & 16,539 & 15,920 & 15,631 & 15,842 & 16,233 & 6,126 & & 17,323 & 3,783 & 19,072 \\
\hline & 545 & 601 & 648 & & & & 1,432 & & 11,465 & & 11,333 & 11,174 & 110 & 11,383 & 11,338 & 12,838 & 12,547 & 13,467 & 313 & 13,375 \\
\hline Norway & 8,316 & 8,285 & 8,363 & 8,254 & 9,504 & 10,523 & 11,603 & 13,634 & 11,222 & 12,557 & 11,509 & 11,979 & 12,369 & 12,109 & 12,543 & 12,119 & 12,243 & 12,395 & 12,119 & 11,889 \\
\hline & . & . & . & . & . & 8,049 & 5,710 & 4,397 & 6,512 & 5,772 & 5,147 & 5,174 & 4,994 & 5,371 & 5,957 & 5,925 & 5,953 & 6,424 & 6,156 & 5,867 \\
\hline Portugal & . & . & . & . & . & . & 5,442 & 5,613 & 5,578 & 5,761 & 5,850 & 5,811 & 5,849 & 5,792 & 6,115 & 6,408 & 6,502 & 6,618 & 6,928 & 7,250 \\
\hline 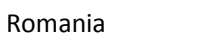 & . & . & . & & . & . & & & & & . & & & & . & . & & 1,777 & 1,568 & 1,606 \\
\hline Slovakia & . & . & $\cdot$ & 7,371 & 5,356 & 4,888 & 5,238 & 5,715 & 5,623 & 4,801 & 4,444 & & 4,351 & 3,419 & 2,541 & 2,608 & 2,714 & 147 & 696 & 3,990 \\
\hline & . & . & . & $\cdot$ & . & . & . & 16,231 & 14,299 & 13,760 & 11,071 & & 12,445 & 11,548 & 11,048 & 11,239 & 11,221 & 11,282 & 11,672 & 11,552 \\
\hline Spain & 17,681 & 16,222 & 4,343 & 1,790 & 10,229 & 9,699 & 9,351 & 8,752 & 8,400 & 8,250 & 8,414 & 8,758 & 8,531 & 8,578 & 8,619 & 8,573 & 8,420 & 8,512 & 8,472 & 8,922 \\
\hline Sweden & 12,110 & 10,473 & 11,924 & 11,297 & 11,236 & 11,007 & 11,108 & 9,678 & 9,310 & 9,239 & 9,476 & 9,205 & 9,586 & 9,732 & 10,260 & 10,322 & 10,771 & 10,832 & 10,755 & 10,723 \\
\hline & 11,712 & 11,755 & 11,833 & 11,550 & 11,689 & 11,788 & 11,752 & 11,687 & 12,712 & 14,223 & 14,135 & 14,278 & 14,586 & 14,742 & 14,879 & 12,834 & 12,776 & 12,760 & 12,533 & 12,990 \\
\hline & 10,053 & 9,787 & & & & & 9,998 & & & & 11,691 & 12,183 & & 13,217 & 14,049 & 13,146 & 14,220 & 14,619 & 15,063 & 15,396 \\
\hline United States & 12,232 & 11,047 & 11,741 & 11,534 & 11,352 & 11,304 & 11,191 & 10,979 & 10,897 & 10,875 & 10,602 & 10,495 & 10,618 & 10,510 & 10,298 & 10,224 & 9,974 & 9,799 & 9,959 & 9,754 \\
\hline
\end{tabular}


Table 3. Real net minimum income benefit levels for two parent household with two children

\begin{tabular}{|c|c|c|c|c|c|c|c|c|c|c|c|c|c|c|c|c|c|c|c|c|}
\hline & 1990 & 1991 & 992 & 993 & 994 & 1995 & 1996 & 1997 & 1998 & 1999 & 2000 & 2001 & 2002 & 2003 & 2004 & 2005 & 2006 & 2007 & 2008 & 2009 \\
\hline Australia & 17,116 & 8,244 & 826 & 254 & 518 &, 211 & 19,727 & 21,131 & 20,911 & 20,845 & 19,761 & 21,769 & 21,740 & 21,920 & 23,103 & 23,156 & 23,443 & 23,557 & 23,740 & 24,176 \\
\hline Austria & 546 & ,086 & 937 &, 570 & 314 & ,710 & 169 & ,372 & 5,036 & 5,345 & 5,466 & 5,467 & 5,716 & 5,721 & 5,929 & 5,866 & 6,681 & 6,814 & 17,102 & 17,137 \\
\hline Belgium & 808 & 4,688 & 4,988 & 5,418 &, 495 & 5,208 & 6,076 & 5,424 & 15,179 & 15,332 & $.5,133$ & 5,063 & 15,587 & 15,548 & 15,791 & 5,620 & 15,643 & 16,337 & 6,273 & 17,141 \\
\hline B & & & & & & & & & & & & & & & . & & & 4,089 & 659 & 4,567 \\
\hline & 1,698 & 2,570 & 2,792 & 2,716 & 2,911 & 1,451 & 8,193 & 17,861 & 17,540 & 17,409 & 17,023 & 16,712 & 16,409 & 16,165 & 16,330 & 16,637 & 19,135 & 19,108 & 18,566 & 18,656 \\
\hline Cyprus & . & . & . & 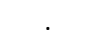 & . & . & . & & & . & . & . & & . & 13,788 & 13,958 & 14,289 & 13,831 & 14,710 & 16,518 \\
\hline Czech Republic & . & . & . & 13,086 & 12,058 & 11,598 & 10,612 & 10,506 & 9,719 & 9,389 & 8,895 & 9,647 & 9,559 & 9,675 & 9,310 & 9,672 & 9,736 & 6,678 & 6,318 & 6,257 \\
\hline enmark & 15,556 & 15,684 & 16,284 & 16,498 & 3,936 & 23,964 & 23,859 & 23,673 & 25,004 & 26,184 & 26,375 & 26,515 & 26,669 & 27,066 & 27,289 & 28,209 & 28,342 & 28,712 & 27,803 & 28,734 \\
\hline & . & . & . & & . & 4,605 & 3,771 & 3,803 & 3,560 & 3,337 & 3,125 & 2,878 & 2,741 & 2,708 & 2,615 & 3,723 & 3,443 & 3,665 & ,602 & 3,689 \\
\hline Finland & 18,814 & 17,357 & 15,033 & 12,985 & 14,173 & 15,278 & 14,873 & 14,566 & 13,974 & 14,077 & 13,636 & 13,671 & 13,781 & 13,967 & 14,319 & 14,555 & 14,646 & 14,536 & 14,164 & 14,657 \\
\hline France & 338 & 9,241 & ,358 & 760 & 976 & 0,117 & 0,348 & 10,270 & 10,339 & 10,863 & 10,895 & 11,080 & 0,967 & 11,022 & 10,953 & 11,213 & 1,225 & 11,392 & 1,389 & 11,687 \\
\hline Germany &. & 12,794 & 12,865 &, 073 & ,984 & 3,186 & 13,009 & 12,562 & 12,634 & 12,647 & 12,897 & 12,945 & 12,869 & 13,011 & 13,092 & 16,192 & 16,324 & 16,352 & 16,194 & 16,566 \\
\hline ing & . & . & 23,296 & 23,630 & 1,318 & 13,510 & 9,563 & 7,247 & 5,921 & 5,307 & 4,730 & 4,269 & 4,128 & 4,268 & 4,332 & 4,243 & 5,604 & 6,194 & 967 & 5,625 \\
\hline Ireland & 15,964 & 15,970 & 15,429 & 14,137 & 4,427 & 15,072 & 15,371 & 16,542 & 15,413 & 15,176 & 14,742 & 14,809 & 14,474 & 16,409 & 16,989 & 18,150 & 19,329 & 20,996 & 127 & 24,707 \\
\hline Italy & 685 & 5,515 & 3,681 & ,349 & ,716 & 5,172 & 15,465 & 15,196 & 14,8 & 17,626 & 17,697 & 18,194 & 110 & 8,815 & 16 & 15 & & & 177 & 23,098 \\
\hline Jap & 13,979 & 14,004 & 14,402 & 4,730 & 4,822 & $.5,504$ & 15,789 & 15,816 & 15,887 & 16,346 & 17,089 & 17,794 & 18,477 & 18,970 & 20,068 & 20,756 & 21,401 & 22,069 & 22,373 & 23,235 \\
\hline & . & . & . & . & . & . & . & . & & . & . & . & & . & 2,975 & 3,055 & 3,05 & 2,833 & ,226 & 3,017 \\
\hline & . & . & . & 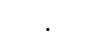 & . & . & . & . & & . & . & . & & . & 3,964 & 3,787 & 3,984 & 4,749 & 5,195 & 6,958 \\
\hline Luxemb & 18,721 & 19,791 & 20,409 & 25,491 & 25,797 & 27,413 & 26,880 & 26,690 & 25,777 & 26,738 & 26,254 & 26,863 & 27,682 & 28,549 & 28,947 & 29,730 & 30,019 & 30,705 & 30,632 & 35,622 \\
\hline Malta & . & . & . & . & . & . & . & & & . & . & . & & 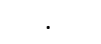 & 11,485 & 12,034 & & 55 & 523 & 12,276 \\
\hline & 7,687 & 7,890 & 8,141 & & 8,555 & & 19,411 & 18,690 & 17,877 & 18,015 & 18,031 & 17,429 & 17,117 & 17,348 & 17,780 & 17,659 & & 18,969 & 0,459 & 20,747 \\
\hline & 012 & & 976 & & & & 665 & & 596 & 692 & 12,446 & 269 & ,201 & 2,485 & 2,440 & 13,718 & 13,652 & 486 & 14,740 & 14,799 \\
\hline Norway & 1,718 & 1,437 & 11,319 & 11,462 & 13,770 & 15,727 & 17,758 & 20,316 & 16,214 & 18,711 & 19,902 & 18,148 & 18,501 & 19,701 & 18,422 & 17,696 & 17,676 & 17,704 & 17,131 & 16,634 \\
\hline Poland & . & . & . & . & . & 8,049 & 5,710 & 4,397 & 8,798 & 7,798 & 6,952 & 5,688 & 5,517 & 5,920 & 5,768 & 5,819 & 5,928 & 6,180 & 6,080 & 5,929 \\
\hline Portugal & . & . & . & 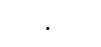 & . & . & 7,840 & 7,971 & 7,922 & 8,187 & 8,314 & 8,254 & 8,307 & 8,233 & 8,669 & 9,107 & 9,249 & 9,411 & 9,699 & 10,168 \\
\hline 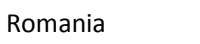 & . & . & . & & . & . & & & & & . & & & & . & . & & 2,148 & 1,896 & 1,930 \\
\hline & . & . & $\cdot$ & 12,286 & 8,927 & 8,146 & 8,151 & 8,436 & 7,959 & 6,568 & 6,076 & 5,675 & 5,946 & 5,045 & 3,916 & 4,020 & 4,205 & 4,919 & 492 & 5,800 \\
\hline & . & . & . & $\cdot$ & . & . & . & 20,259 & 17,727 & & 11,843 & 10,189 & 13,796 & 12,761 & 12,172 & 12,391 & 12,371 & 12,439 & 12,802 & 12,690 \\
\hline Spain & 19,390 & 7,791 & 5,730 & 2,930 & 1,218 & 10,640 & 10,258 & 9,601 & 9,215 & 9,050 & 9,210 & 9,596 & 9,348 & 9,377 & 9,656 & 9,608 & 9,438 & 9,548 & 9,497 & 9,994 \\
\hline Sweden & 15,673 & 14,915 & 15,436 & 14,625 & 14,546 & 14,249 & 14,379 & 12,616 & 12,163 & 12,046 & 12,376 & 12,049 & 12,441 & 12,626 & 13,318 & 13,351 & 13,893 & 13,975 & 13,860 & 13,820 \\
\hline & 14,975 & 15,031 & 15,131 & 14,760 & 4,937 & 15,064 & 14,969 & 14,840 & 15,331 & 16,318 & 16,240 & 16,426 & 16,779 & 16,959 & 17,117 & 14,759 & 14,693 & 14,674 & 14,414 & 14,939 \\
\hline & 12,164 & & & & & & & & & 13,460 & 14,275 & 14,798 & & 16,102 & 16,857 & 15,926 & & 17,462 & 17,873 & 18,272 \\
\hline United States & 14,861 & 14,264 & 14,233 & 13,984 & 13,771 & 13,687 & 13,522 & 13,271 & 13,183 & 13,168 & 12,833 & 12,679 & 12,937 & 12,854 & 12,643 & 12,744 & 12,427 & 12,218 & 12,354 & 12,285 \\
\hline
\end{tabular}


Table 4. Real net minimum income benefit levels (average of the three household types)

\begin{tabular}{|c|c|c|c|c|c|c|c|c|c|c|c|c|c|c|c|c|c|c|c|c|}
\hline & 1990 & 1991 & 1992 & 1993 & 1994 & 1995 & 1996 & 1997 & 1998 & 1999 & 2000 & 2001 & 2002 & 2003 & 2004 & 2005 & 2006 & 2007 & 2008 & 2009 \\
\hline Australia & 12,231 & 12,847 & 256 & 544 & 687 & 13,524 & 13,885 & 14,855 & 14,685 & 14,633 & 13,870 & 15,229 & 15,210 & 15,332 & 16,144 & 16,163 & 16,376 & 16,455 & 16,569 & 16,874 \\
\hline Austria & 545 & ,211 & ,866 & ,322 & 188, & 1,484 & L,109 & , 996 & D,865 & 1,226 & 11,311 & 11,308 & 1,496 & 1,486 & 1,645 & 1,601 & 2,034 & 2,105 & 12,327 & 12,398 \\
\hline Belgium & 452 & 2,458 & 2,656 & 3,020 & 3,094 & 3,696 & 3,584 & 13,017 & 12,845 & 12,964 & 12,811 & 12,752 & 13,220 & 13,201 & 13,422 & 13,261 & 13,231 & 13,818 & 13,998 & 14,861 \\
\hline Bulga & & & & & 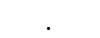 & & & & & & 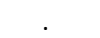 & & & & . &. & & 3,013 & 2,682 & 3,205 \\
\hline Canada & 6,311 & 16,985 & 17,210 & 17,155 & 17,389 & 16,323 & 13,761 & 13,542 & 13,349 & 13,301 & 13,072 & 12,881 & 12,679 & 12,508 & 12,667 & 12,851 & 14,345 & 14,356 & 14,010 & 13,924 \\
\hline Cyprus & . & . & . & . & . & . & . & & & . & . & . & & . & 10,145 & 10,270 & 10,516 & 10,176 & 10,833 & 12,161 \\
\hline Czech Republic & . & . & . & 9,160 & 8,437 & 8,120 & 7,451 & 7,389 & 6,895 & 6,660 & 6,310 & 6,923 & 6,860 & 6,943 & 6,681 & 6,967 & 7,023 & 4,520 & 4,276 & 4,234 \\
\hline Denmark & 10,806 & 10,921 & 11,363 & 11,512 & 15,247 & 15,777 & 15,728 & 15,625 & 16,149 & 16,581 & 16,594 & 16,692 & 16,796 & 17,065 & 17,363 & 17,955 & 18,028 & 18,262 & 17,684 & 18,247 \\
\hline & . & . & . & . & . & 3,160 & 2,588 & 2,610 & 2,443 & 2,290 & 2,145 & 1,975 & 1,881 & 1,859 & 1,795 & 2,555 & 2,363 & 2,515 & ,472 & 2,604 \\
\hline Finland & 13,158 & 12,140 & 10,513 & 9,081 & 9,912 & 10,698 & 10,414 & 10,199 & 9,784 & 9,857 & 9,548 & 9,573 & 9,649 & 9,780 & 10,027 & 10,192 & 10,255 & 10,179 & 9,918 & 10,263 \\
\hline France & 7,195 & 120 & 211 & 520 & 587 & 7,796 & 7,974 & ,912 & 7,967 & 8,363 & 8,474 & 8,549 & 8,529 & 8,573 & 8,520 & 8,721 & 8,731 & 860 & ,858 & 9,090 \\
\hline Germany & . & 9,239 & 9,384 & 9,686 & 625 & 9,767 & 9,634 & 9,306 & 9,355 & 9,453 & 9,640 & 9,676 & 9,631 & 9,738 & 9,798 & 11,724 & 11,820 & 11,840 & 11,737 & 11,998 \\
\hline unga & . & . & 15,898 & 16,157 & 14,502 & 9,188 & 6,508 & 4,936 & 4,035 & 3,618 & 3,220 & 2,903 & 2,804 & 2,907 & 2,950 & 2,889 & 3,793 & 4,949 &, 666 & 4,399 \\
\hline Ireland & 11,434 & 11,479 & 11,219 & 10,150 & 10,386 & 10,889 & 11,123 & 11,990 & 11,302 & 11,042 & 10,680 & 10,677 & 10,759 & 11,716 & 12,140 & 12,986 & 13,836 & 15,022 & 15,831 & 17,680 \\
\hline Italy & 735 & 18,878 & 17,521 & 14,317 & ,103 & 11,223 & 11,440 & 11,241 & 10,958 & 13,037 & 13,096 & 13,458 &, 618 & 3,919 & 14,290 & 14,882 & & & & 17,092 \\
\hline pan & 11,174 & 11,179 & 11,453 & 11,691 & 1,775 & 12,403 & 12,631 & 12,585 & 12,684 & 13,076 & 13,671 & 14,235 & 14,782 & 15,174 & 15,995 & 16,659 & 17,172 & 17,708 & 17,952 & 18,643 \\
\hline & . & . & . & . & . & . & . & . & . & . & . & . & & . & 1,983 & 2,036 & 2,060 & 1,889 & ,484 & 2,012 \\
\hline & . & . & . & . & . & . & . & . & 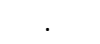 & . & . & . & & . & 2,643 & 2,525 & 2,656 & 3,166 & 3,463 & 4,639 \\
\hline Luxemb & 13,877 & 14,682 & 15,154 & 18,843 & 19,069 & 20,275 & 19,876 & 19,750 & 19,119 & 19,779 & 19,422 & 19,885 & 20,439 & 21,095 & 21,389 & 21,977 & 22,191 & 22,720 & 22,678 & 27,923 \\
\hline Malta & . & . & . & . & . & . & . & & & . & . & . & & . & 9,607 & 10,318 & 10,555 & 9,878 & & 10,264 \\
\hline & 14,723 & 14,894 & 15,104 & 15,634 & 15,457 & 16,572 & 16,091 & 15,389 & 14,891 & 15,010 & 14,998 & 14,639 & 14,378 & 14,573 & 14,940 & 14,841 & 15,430 & 15,944 & ,996 & 17,179 \\
\hline & 096 & & & 9,462 & & & & 9,875 & & & 9,736 & 9,606 & 9,561 & 9,805 & 9,774 & 10,854 & 10,744 & 11,348 & 11,380 & 11,417 \\
\hline Norway & 9,250 & 9,118 & 9,112 & 9,082 & 10,582 & 11,821 & 13,126 & 15,195 & 12,298 & 13,995 & 13,938 & 13,532 & 13,841 & 14,171 & 13,894 & 13,507 & 13,526 & 13,580 & 13,172 & 12,819 \\
\hline & . & . & . & . & . & 7,650 & 5,427 & 4,179 & 6,298 & 5,582 & 4,976 & 4,612 & 4,496 & 4,769 & 4,874 & 4,875 & 4,925 & 5,171 & 5,008 & 4,818 \\
\hline Portugal & . & . & . & . & . & . & 5,226 & 5,314 & 5,281 & 5,458 & 5,542 & 5,503 & 5,538 & 5,489 & 5,779 & 6,071 & 6,166 & 6,274 & 6,475 & 6,787 \\
\hline . & . & . & . & & . & . & & & & & . & & & & . & . & & 1,509 & 1,331 & 1,353 \\
\hline Slovakia & . & . & . & 7,917 & 5,753 & 5,250 & 5,414 & 5,739 & & 4,631 & 4,285 & 4,002 & 4,193 & 3,363 & 2,671 & 2,742 & 2,865 & 3,350 & 754 & 3,971 \\
\hline & . & . & . &. &. & . & . & 13,680 & 11,978 & 11,282 & 8,673 & 7,454 & 10,191 & 9,420 & 8,979 & 9,142 & & 9,177 & 9,434 & 9,354 \\
\hline Spain & 16,153 & 14,821 & 3,104 & 10,772 & 9,346 & 8,870 & 8,552 & 8,004 & 7,682 & 7,545 & 7,643 & 7,980 & 7,777 & 7,759 & 7,823 & 7,786 & 7,655 & 7,747 & 7,717 & 8,134 \\
\hline Sweden & 11,081 & 10,190 & 10,907 & 10,333 & 10,278 & 10,068 & 10,160 & 8,873 & 8,622 & 8,530 & 8,756 & 8,514 & 8,795 & 8,935 & 9,416 & 9,468 & 9,825 & 9,878 & 9,804 & 9,775 \\
\hline & 11,145 & 11,186 & 11,260 & 10,993 & 11,125 & 11,219 & 11,175 & 11,105 & 11,710 & 12,723 & 12,654 & 12,793 & 13,068 & 13,208 & 13,331 & 11,497 & 11,445 & 11,431 & 11,228 & 11,637 \\
\hline & 9,006 & & 9,099 & 9,085 & 9,240 & 9,032 & 8,937 & 9,294 & 9,462 & 9,784 & 10,341 & 10,688 & 11,318 & 11,475 & 12,040 & 11,413 & 12,125 & 12,456 & 12,720 & 13,001 \\
\hline United States & 9,623 & 9,039 & 9,276 & 9,106 & 8,965 & 8,920 & 8,830 & 8,667 & 8,612 & 8,161 & 7,956 & 7,868 & 7,998 & 7,936 & 7,793 & 7,805 & 7,614 & 7,485 & 7,585 & 7,497 \\
\hline
\end{tabular}


Table 5. Net minimum income replacement rate for single person household without children

\begin{tabular}{|c|c|c|c|c|c|c|c|c|c|c|c|c|c|c|c|c|c|c|c|c|}
\hline & 1990 & 1991 & 1992 & 1993 & 1994 & 1995 & 1996 & 1997 & 1998 & 1999 & 2000 & 2001 & 2002 & 2003 & 2004 & 2005 & 2006 & 2007 & 2008 & 2009 \\
\hline Australia & 30 & 31 & 32 & 31 & 29 & 29 & 30 & 30 & 29 & 29 & 27 & 27 & 26 & 25 & 25 & 25 & 25 & 24 & 23 & 22 \\
\hline Austria & 25 & 25 & 27 & 27 & 27 & 27 & 27 & 27 & 27 & 27 & 27 & 27 & 27 & 27 & 27 & 26 & 27 & 26 & 26 & 27 \\
\hline Belgium & 38 & 38 & 37 & 38 & 38 & 39 & 39 & 38 & 39 & 38 & 38 & 38 & 39 & 38 & 38 & 36 & 35 & 35 & 36 & 37 \\
\hline Bulgaria & . & $\cdot$ & $\cdot$ & $\cdot$ & $\cdot$ & $\cdot$ & $\cdot$ & $\cdot$ & . & . & . & $\cdot$ & $\cdot$ & . & . & . & . & 30 & 24 & 26 \\
\hline Canada & 34 & 37 & 37 & 36 & 36 & 34 & 28 & 27 & 27 & 27 & 26 & 24 & 23 & 23 & 23 & 22 & 21 & 21 & 21 & 21 \\
\hline Cyprus & . & . & $\cdot$ & . & . & . & . & & . & . & . & . & & . & 26 & 26 & 26 & 24 & . & . \\
\hline Czech Republic & . & . & . & 40 & 37 & 36 & 33 & 34 & 35 & 32 & 32 & 36 & 34 & 33 & 30 & 30 & 28 & 15 & 14 & 14 \\
\hline Denmark & 30 & 30 & 30 & 30 & 40 & 45 & 43 & 43 & 41 & 42 & 42 & 42 & 41 & 40 & 40 & 39 & 39 & 38 & 36 & 38 \\
\hline Estonia & . & . & . & . & . & 17 & 17 & 17 & 16 & 16 & 14 & 13 & 12 & 11 & 10 & 13 & 11 & 11 & 10 & 11 \\
\hline Finland & 32 & 30 & 31 & 32 & 31 & 30 & 28 & 27 & 27 & 25 & 25 & 24 & 23 & 23 & 22 & 22 & 21 & 21 & 20 & 20 \\
\hline France & 29 & 29 & 28 & 29 & 29 & 29 & 30 & 28 & 28 & 28 & 28 & 28 & 28 & 27 & 27 & 27 & 27 & 26 & 26 & 26 \\
\hline Germany & 20 & 21 & 21 & 21 & 21 & 21 & 21 & 21 & 21 & 21 & 21 & 20 & 20 & 20 & 20 & 23 & 23 & 23 & 22 & 23 \\
\hline Hungary & . & . & 28 & 37 & 43 & 41 & 36 & 30 & 27 & 25 & 22 & 24 & 22 & 21 & 22 & 20 & 23 & 31 & 25 & 24 \\
\hline Ireland & 33 & 34 & 33 & 31 & 31 & 32 & 32 & 32 & 32 & 31 & 29 & 28 & 29 & 30 & 30 & 32 & 34 & 34 & 35 & 36 \\
\hline Italy & 33 & 34 & 34 & 35 & 32 & 31 & 29 & 28 & 28 & 34 & 34 & 35 & 36 & 36 & 36 & 37 & 38 & 39 & 39 & 40 \\
\hline Japan & 27 & 27 & 28 & 28 & 28 & 28 & 27 & 27 & 28 & 29 & 29 & 28 & 29 & 29 & 29 & 29 & 29 & 29 & 29 & 30 \\
\hline Latvia & . & . & . & . & . & . & . & . & . & . & . & . & . & . & 14 & 15 & 14 & 12 & 9 & 12 \\
\hline Lithuania & . & . & . & . & . & . & . & . & . & . & . & . & . & . & 15 & 14 & 14 & 14 & 16 & 21 \\
\hline Luxembourg & 36 & 36 & 38 & 45 & 45 & 46 & 45 & 47 & 44 & 45 & 44 & 44 & 42 & 44 & 45 & 45 & 45 & 46 & 45 & 53 \\
\hline Malta & . & . & . & . & . & . & . & . & . & . & . & . & . & . & 38 & 43 & 43 & 37 & 37 & 37 \\
\hline Netherlands & 46 & 47 & 48 & 47 & 46 & 48 & 47 & 44 & 43 & 44 & 43 & 43 & 40 & 40 & 40 & 39 & 39 & 40 & 40 & 39 \\
\hline New Zealand & 30 & 26 & 26 & 26 & 26 & 26 & 25 & 25 & 24 & 24 & 25 & 24 & 24 & 24 & 24 & 26 & 26 & 25 & 25 & 24 \\
\hline Norway & 39 & 37 & 35 & 35 & 38 & 40 & 42 & 48 & 39 & 43 & 43 & 44 & 43 & 43 & 41 & 39 & 38 & 36 & 35 & 35 \\
\hline Poland & . & . & . & . & . & 57 & 45 & 36 & 37 & 33 & 31 & 32 & 32 & 31 & 30 & 29 & 28 & 26 & 24 & 23 \\
\hline Portugal & . & . & . & . & . & . & 23 & 22 & 23 & 24 & 24 & 24 & 24 & 23 & 23 & 24 & 24 & 24 & 24 & 24 \\
\hline Romania & . & . & . & . & . & . & . & & . & . & . & . & & . & . & . & . & 15 & 13 & 13 \\
\hline Slovakia & . & . & . & 37 & 32 & 31 & 32 & 34 & 35 & 38 & 32 & 34 & 34 & 25 & 24 & 22 & 22 & 23 & 22 & 20 \\
\hline Slovenia & . & . & . & . & . & . & . & 25 & 23 & 21 & 21 & 19 & 36 & 34 & 30 & 29 & 29 & 27 & 27 & 27 \\
\hline Spain & 38 & 35 & 33 & 31 & 30 & 30 & 28 & 27 & 26 & 25 & 26 & 27 & 27 & 26 & 25 & 25 & 25 & 25 & 24 & 25 \\
\hline Sweden & 34 & 32 & 32 & 33 & 32 & 32 & 31 & 27 & 26 & 25 & 25 & 24 & 24 & 25 & 25 & 24 & 23 & 23 & 22 & 21 \\
\hline Switzerland & 26 & 26 & 27 & 26 & 26 & 26 & 26 & 26 & 27 & 28 & 28 & 28 & 27 & 27 & 27 & 22 & 21 & 21 & 21 & 20 \\
\hline United Kingdom & 22 & 23 & 23 & 23 & 24 & 23 & 23 & 22 & 22 & 21 & 21 & 20 & 20 & 20 & 20 & 20 & 19 & 19 & 19 & 20 \\
\hline United States & 7 & 7 & 7 & 7 & 7 & 7 & 7 & 7 & 7 & 2 & 2 & 2 & 2 & 2 & 2 & 2 & 2 & 2 & 2 & 2 \\
\hline
\end{tabular}


Table 6. Net minimum income replacement rate for lone parent household with two children

\begin{tabular}{|c|c|c|c|c|c|c|c|c|c|c|c|c|c|c|c|c|c|c|c|c|}
\hline & 1990 & 1991 & 1992 & 1993 & 1994 & 1995 & 1996 & 1997 & 1998 & 1999 & 2000 & 2001 & 2002 & 2003 & 2004 & 2005 & 2006 & 2007 & 2008 & 2009 \\
\hline Australia & 48 & 48 & 49 & 49 & 47 & 47 & 48 & 49 & 48 & 48 & 46 & 50 & 48 & 47 & 48 & 48 & 48 & 45 & 45 & 43 \\
\hline Austria & 48 & 48 & 49 & 49 & 48 & 49 & 48 & 48 & 48 & 48 & 47 & 47 & 48 & 47 & 48 & 47 & 48 & 47 & 48 & 48 \\
\hline Belgium & 53 & 52 & 52 & 53 & 53 & 53 & 54 & 53 & 52 & 52 & 52 & 52 & 53 & 52 & 52 & 52 & 51 & 50 & 52 & 54 \\
\hline Bulgaria & & . & . & & . & & . & . & & . & & . & & . & . & & . & 79 & 67 & 71 \\
\hline Canada & 70 & 75 & 74 & 74 & 74 & 70 & 59 & 58 & 58 & 57 & 56 & 54 & 52 & 51 & 52 & 50 & 57 & 55 & 52 & 51 \\
\hline Cyprus & . & . & . & . & . & . & . & . & . & . & . & . & . & . & 47 & 46 & 46 & 41 & . & . \\
\hline Czech Republic & & . & . & 81 & 76 & 77 & 72 & 74 & 67 & 62 & 61 & 69 & 65 & 63 & 60 & 57 & 54 & 29 & 27 & 26 \\
\hline Denmark & 56 & 56 & 57 & 57 & 62 & 60 & 59 & 58 & 57 & 59 & 57 & 57 & 56 & 56 & 56 & 56 & 55 & 54 & 52 & 53 \\
\hline Estonia & . & . & . & . & . & 38 & 37 & 39 & 37 & 36 & 31 & 28 & 26 & 24 & 21 & 28 & 24 & 24 & 24 & 27 \\
\hline Finland & 63 & 59 & 60 & 61 & 62 & 57 & 55 & 53 & 52 & 50 & 49 & 48 & 47 & 46 & 45 & 44 & 43 & 43 & 41 & 42 \\
\hline France & 42 & 42 & 42 & 42 & 42 & 42 & 44 & 40 & 41 & 41 & 43 & 42 & 42 & 42 & 42 & 42 & 42 & 41 & 41 & 41 \\
\hline Germany & 41 & 41 & 42 & 44 & 44 & 43 & 41 & 41 & 40 & 39 & 38 & 37 & 37 & 37 & 36 & 42 & 41 & 40 & 40 & 40 \\
\hline Hungary & . & . & 50 & 59 & 61 & 57 & 54 & 47 & 43 & 39 & 33 & 33 & 30 & 31 & 31 & 30 & 41 & 67 & 66 & 63 \\
\hline Ireland & 46 & 47 & 46 & 44 & 43 & 46 & 45 & 45 & 44 & 41 & 38 & 37 & 37 & 41 & 41 & 42 & 45 & 46 & 46 & 48 \\
\hline Italy & 64 & 64 & 65 & 66 & 62 & 59 & 55 & 52 & 50 & 61 & 61 & 63 & 65 & 66 & 67 & 68 & 70 & 71 & 71 & 73 \\
\hline Japan & 68 & 66 & 68 & 68 & 67 & 70 & 67 & 67 & 67 & 70 & 71 & 69 & 72 & 70 & 72 & 73 & 73 & 72 & 72 & 75 \\
\hline Latvia & . & . & . & . & . & . & . & . & . & . & . & . & . & . & 37 & 37 & 36 & 30 & 23 & 31 \\
\hline Lithuania & . & . & . & . & . & . & . & . & . & . & . & . & . & . & 42 & 37 & 37 & 39 & 40 & 56 \\
\hline Luxembourg & 45 & 46 & 48 & 58 & 57 & 59 & 58 & 59 & 55 & 56 & 55 & 56 & 56 & 58 & 58 & 58 & 58 & 59 & 57 & 77 \\
\hline Malta & . & . & . & . & . & . & . & . & . & . & . & . & . & . & 53 & 55 & 55 & 52 & 55 & 55 \\
\hline Netherlands & 63 & 66 & 64 & 62 & 61 & 64 & 62 & 59 & 57 & 58 & 59 & 56 & 52 & 52 & 52 & 51 & 50 & 52 & 55 & 55 \\
\hline New Zealand & 58 & 52 & 52 & 53 & 56 & 55 & 53 & 51 & 49 & 49 & 49 & 49 & 48 & 49 & 49 & 50 & 44 & 44 & 44 & 43 \\
\hline Norway & 33 & 32 & 32 & 31 & 35 & 38 & 41 & 47 & 39 & 43 & 41 & 44 & 44 & 43 & 42 & 39 & 38 & 38 & 37 & 38 \\
\hline Poland & . & . & . & . & . & 61 & 48 & 39 & 60 & 55 & 52 & 52 & 53 & 51 & 57 & 57 & 55 & 53 & 48 & 45 \\
\hline Portugal & . & . & . & & . & & 46 & 48 & 49 & 50 & 51 & 50 & 50 & 49 & 50 & 52 & 51 & 51 & 52 & 52 \\
\hline Romania & . & . & . & & . & . & . & . & . & . & & . & . & . & . & . & . & 38 & 35 & 37 \\
\hline Slovakia & . & . & . & 56 & 50 & 48 & 52 & 55 & 58 & 61 & 58 & 56 & 59 & 47 & 31 & 29 & 29 & 29 & 31 & 31 \\
\hline Slovenia & . & . & . & . & . & . & . & 75 & 70 & 71 & 63 & 60 & 88 & 84 & 74 & 72 & 70 & 68 & 70 & 69 \\
\hline Spain & 55 & 51 & 47 & 45 & 43 & 42 & 40 & 38 & 37 & 34 & 36 & 38 & 38 & 37 & 38 & 38 & 38 & 38 & 36 & 37 \\
\hline Sweden & 65 & 56 & 62 & 63 & 63 & 63 & 62 & 54 & 50 & 49 & 47 & 45 & 47 & 47 & 48 & 46 & 46 & 45 & 43 & 42 \\
\hline Switzerland & 40 & 40 & 41 & 39 & 38 & 39 & 38 & 38 & 41 & 45 & 45 & 44 & 44 & 43 & 43 & 36 & 35 & 34 & 34 & 33 \\
\hline United Kingdom & 41 & 42 & 43 & 44 & 45 & 44 & 43 & 42 & 42 & 43 & 43 & 42 & 44 & 44 & 45 & 42 & 45 & 44 & 46 & 48 \\
\hline United States & 44 & 40 & 43 & 42 & 41 & 41 & 40 & 39 & 38 & 36 & 36 & 35 & 34 & 33 & 33 & 32 & 31 & 31 & 31 & 29 \\
\hline
\end{tabular}


Table 7. Net minimum income replacement rate for two parent household with two children

\begin{tabular}{|c|c|c|c|c|c|c|c|c|c|c|c|c|c|c|c|c|c|c|c|c|}
\hline & 1990 & 1991 & 1992 & 1993 & 1994 & 1995 & 1996 & 1997 & 1998 & 1999 & 2000 & 2001 & 2002 & 2003 & 2004 & 2005 & 2006 & 2007 & 2008 & 2009 \\
\hline Australia & 66 & 68 & 70 & 69 & 67 & 66 & 67 & 68 & 66 & 66 & 64 & 68 & 65 & 63 & 63 & 64 & 64 & 61 & 59 & 57 \\
\hline Austria & 58 & 58 & 60 & 60 & 59 & 59 & 59 & 61 & 59 & 58 & 57 & 57 & 58 & 57 & 58 & 58 & 60 & 59 & 60 & 59 \\
\hline Belgium & 53 & 52 & 52 & 53 & 53 & 53 & 54 & 53 & 52 & 52 & 52 & 52 & 53 & 52 & 52 & 52 & 51 & 50 & 50 & 51 \\
\hline Bulgaria & . & . & . & . & . & . & . & . & . & . & . & . & . & . & . & . & . & 87 & 73 & 85 \\
\hline Canada & 79 & 85 & 84 & 83 & 83 & 78 & 66 & 64 & 64 & 62 & 61 & 57 & 55 & 54 & 54 & 53 & 61 & 58 & 56 & 56 \\
\hline Cyprus & . & . & . & . & . & . & . & . & . & . & . & . & . & . & 59 & 58 & 58 & 52 & . & . \\
\hline Czech Republic & . & . & . & 104 & 98 & 99 & 93 & 95 & 85 & 79 & 78 & 86 & 81 & 79 & 75 & 71 & 67 & 40 & 37 & 36 \\
\hline Denmark & 74 & 74 & 74 & 74 & 103 & 98 & 96 & 94 & 96 & 103 & 102 & 102 & 101 & 100 & 98 & 98 & 96 & 94 & 91 & 94 \\
\hline Estonia & . & . & . & . & $\cdot$ & 49 & 49 & 51 & 48 & 47 & 41 & 37 & 34 & 31 & 28 & 37 & 31 & 32 & 32 & 33 \\
\hline Finland & 81 & 76 & 78 & 79 & 80 & 74 & 72 & 69 & 69 & 66 & 64 & 63 & 62 & 61 & 58 & 58 & 57 & 56 & 54 & 55 \\
\hline France & 51 & 50 & 50 & 51 & 51 & 50 & 53 & 49 & 49 & 50 & 50 & 50 & 50 & 49 & 49 & 49 & 49 & 48 & 48 & 47 \\
\hline Germany & 49 & 50 & 50 & 50 & 50 & 49 & 46 & 46 & 46 & 44 & 42 & 41 & 41 & 41 & 40 & 50 & 49 & 49 & 48 & 48 \\
\hline Hungary & . & . & 67 & 83 & 91 & 85 & 79 & 69 & 62 & 56 & 48 & 49 & 46 & 45 & 47 & 45 & 58 & 65 & 64 & 61 \\
\hline Ireland & 65 & 65 & 63 & 61 & 60 & 63 & 62 & 62 & 59 & 56 & 53 & 52 & 50 & 57 & 57 & 59 & 63 & 64 & 65 & 68 \\
\hline Italy & 76 & 77 & 78 & 79 & 74 & 71 & 67 & 62 & 61 & 73 & 73 & 75 & 78 & 79 & 80 & 82 & 84 & 85 & 85 & 88 \\
\hline Japan & 67 & 66 & 68 & 69 & 67 & 69 & 66 & 66 & 67 & 69 & 70 & 69 & 71 & 70 & 72 & 71 & 71 & 71 & 71 & 73 \\
\hline Latvia & . & . & . & . & . & . & . & . & . & . & . & . & . & . & 49 & 49 & 48 & 40 & 31 & 41 \\
\hline Lithuania & . & . & . & $\cdot$ & . & . & . & . & $\cdot$ & $\cdot$ & $\cdot$ & . & & . & 56 & 49 & 49 & 52 & 55 & 75 \\
\hline Luxembourg & 59 & 60 & 63 & 75 & 74 & 76 & 75 & 77 & 72 & 72 & 72 & 73 & 72 & 75 & 75 & 76 & 75 & 77 & 74 & 87 \\
\hline Malta & . & . & . & . & . & . & . & . & . & . & . & . & . & . & 57 & 58 & 59 & 55 & 58 & 58 \\
\hline Netherlands & 69 & 72 & 70 & 68 & 67 & 70 & 68 & 65 & 62 & 63 & 64 & 61 & 57 & 57 & 57 & 56 & 55 & 57 & 59 & 60 \\
\hline New Zealand & 65 & 59 & 58 & 60 & 62 & 61 & 58 & 56 & 54 & 54 & 54 & 53 & 53 & 54 & 54 & 54 & 48 & 48 & 49 & 47 \\
\hline Norway & 47 & 45 & 43 & 43 & 51 & 56 & 62 & 70 & 56 & 64 & 71 & 66 & 65 & 69 & 62 & 57 & 55 & 54 & 52 & 53 \\
\hline Poland & . & $\cdot$ & . & $\cdot$ & . & 61 & 48 & 39 & 81 & 74 & 70 & 57 & 58 & 56 & 55 & 56 & 55 & 51 & 47 & 46 \\
\hline Portugal & . & . & . & . & . & . & 67 & 68 & 70 & 71 & 72 & 71 & 70 & 69 & 71 & 73 & 73 & 72 & 73 & 73 \\
\hline Romania & . & . & . & . & . & . & . & . & . & . & . & . & . & . & . & . & . & 46 & 42 & 45 \\
\hline Slovakia & . & . & . & 94 & 83 & 80 & 81 & 81 & 83 & 84 & 80 & 77 & 80 & 69 & 48 & 45 & 45 & 46 & 47 & 45 \\
\hline Slovenia & . & . & . & . & . & . & . & 93 & 87 & 86 & 68 & 63 & 97 & 93 & 81 & 79 & 77 & 74 & 76 & 76 \\
\hline Spain & 60 & 56 & 51 & 49 & 47 & 47 & 44 & 42 & 41 & 38 & 40 & 42 & 41 & 41 & 43 & 42 & 43 & 42 & 41 & 41 \\
\hline Sweden & 84 & 80 & 80 & 82 & 81 & 82 & 80 & 70 & 65 & 63 & 61 & 60 & 61 & 61 & 62 & 60 & 59 & 58 & 56 & 54 \\
\hline Switzerland & 51 & 51 & 52 & 49 & 49 & 50 & 49 & 49 & 49 & 52 & 51 & 51 & 51 & 50 & 49 & 41 & 41 & 39 & 39 & 38 \\
\hline United Kingdom & 50 & 51 & 52 & 53 & 54 & 53 & 52 & 50 & 51 & 53 & 52 & 51 & 53 & 53 & 54 & 51 & 53 & 53 & 55 & 57 \\
\hline United States & 54 & 52 & 52 & 51 & 49 & 49 & 48 & 47 & 46 & 44 & 43 & 42 & 42 & 41 & 40 & 40 & 39 & 39 & 39 & 37 \\
\hline
\end{tabular}


Table 8. Net minimum income replacement rate (average of the three household types)

\begin{tabular}{|c|c|c|c|c|c|c|c|c|c|c|c|c|c|c|c|c|c|c|c|c|}
\hline & 1990 & 1991 & 1992 & 1993 & 1994 & 1995 & 1996 & 1997 & 1998 & 1999 & 2000 & 2001 & 2002 & 2003 & 2004 & 2005 & 2006 & 2007 & 2008 & 2009 \\
\hline Australia & 48 & 49 & 50 & 50 & 48 & 48 & 48 & 49 & 48 & 47 & 46 & 48 & 46 & 45 & 45 & 45 & 46 & 43 & 42 & 41 \\
\hline Austria & 43 & 43 & 45 & 45 & 45 & 45 & 45 & 45 & 45 & 44 & 43 & 44 & 44 & 44 & 44 & 44 & 45 & 44 & 45 & 44 \\
\hline Belgium & 48 & 47 & 47 & 48 & 48 & 49 & 49 & 48 & 48 & 48 & 48 & 47 & 48 & 48 & 48 & 46 & 46 & 45 & 46 & 47 \\
\hline Bulgaria & . & . & . & . & . & . & . & . & . & . & . & . & . & . & . & . & . & 65 & 55 & 61 \\
\hline Canada & 61 & 65 & 65 & 65 & 64 & 61 & 51 & 50 & 50 & 49 & 48 & 45 & 44 & 43 & 43 & 42 & 46 & 45 & 43 & 43 \\
\hline Cyprus & . & . & $\cdot$ & . & 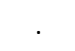 & . & . & . & . & . & . & . & . & . & 44 & 43 & 43 & 39 & . & . \\
\hline Czech Republic & . & . & . & 75 & 70 & 71 & 66 & 68 & 62 & 58 & 57 & 64 & 60 & 58 & 55 & 53 & 50 & 28 & 26 & 25 \\
\hline Denmark & 53 & 53 & 54 & 54 & 68 & 67 & 66 & 65 & 65 & 68 & 67 & 67 & 66 & 65 & 65 & 65 & 63 & 62 & 60 & 62 \\
\hline Estonia & . & . & . & . & . & 35 & 34 & 36 & 34 & 33 & 28 & 26 & 24 & 22 & 20 & 26 & 22 & 22 & 22 & 24 \\
\hline Finland & 59 & 55 & 56 & 57 & 58 & 53 & 52 & 50 & 49 & 47 & 46 & 45 & 44 & 43 & 42 & 41 & 40 & 40 & 38 & 39 \\
\hline France & 41 & 40 & 40 & 41 & 41 & 40 & 42 & 39 & 39 & 40 & 41 & 40 & 40 & 40 & 39 & 39 & 39 & 38 & 38 & 38 \\
\hline Germany & 37 & 37 & 38 & 39 & 38 & 38 & 36 & 36 & 36 & 34 & 34 & 33 & 33 & 33 & 32 & 38 & 38 & 37 & 37 & 37 \\
\hline Hungary & . & . & 48 & 60 & 65 & 61 & 56 & 49 & 44 & 40 & 34 & 35 & 33 & 32 & 34 & 32 & 41 & 54 & 51 & 49 \\
\hline Ireland & 48 & 49 & 47 & 45 & 45 & 47 & 46 & 46 & 45 & 43 & 40 & 39 & 39 & 43 & 42 & 44 & 47 & 48 & 48 & 51 \\
\hline Italy & 58 & 58 & 59 & 60 & 56 & 54 & 50 & 47 & 46 & 56 & 56 & 58 & 60 & 60 & 61 & 62 & 64 & 65 & 65 & 67 \\
\hline Japan & 54 & 53 & 55 & 55 & 54 & 56 & 53 & 53 & 54 & 56 & 56 & 55 & 57 & 56 & 58 & 57 & 58 & 57 & 57 & 60 \\
\hline Latvia & . & . & . & . & . & . & . & . & . & . & . & . & . & . & 33 & 34 & 32 & 27 & 21 & 28 \\
\hline Lithuania & . & . & . & . & . & . & . & . & . & . & . & . & . & . & 38 & 33 & 33 & 35 & 37 & 51 \\
\hline Luxembourg & 47 & 48 & 50 & 59 & 58 & 60 & 60 & 61 & 57 & 57 & 57 & 57 & 57 & 59 & 59 & 60 & 59 & 60 & 59 & 72 \\
\hline Malta & . & . & . & . & . & . & . & . & . & . & . & . & . & . & 49 & 52 & 52 & 48 & 50 & 50 \\
\hline Netherlands & 59 & 62 & 60 & 59 & 58 & 61 & 59 & 56 & 54 & 55 & 55 & 53 & 50 & 50 & 50 & 49 & 48 & 49 & 51 & 52 \\
\hline New Zealand & 51 & 46 & 45 & 46 & 48 & 47 & 45 & 44 & 42 & 42 & 42 & 42 & 42 & 42 & 42 & 43 & 39 & 39 & 40 & 38 \\
\hline Norway & 40 & 38 & 37 & 36 & 42 & 44 & 48 & 55 & 45 & 50 & 52 & 51 & 51 & 52 & 49 & 45 & 44 & 43 & 41 & 42 \\
\hline Poland & . & . & . & . & . & 60 & 47 & 38 & 59 & 54 & 51 & 47 & 48 & 46 & 48 & 48 & 46 & 43 & 40 & 38 \\
\hline Portugal & . & . & . & . & . & . & 45 & 46 & 48 & 48 & 49 & 48 & 48 & 47 & 48 & 50 & 49 & 49 & 50 & 50 \\
\hline Romania & . & . & . & . & & . & . & . & . & . & . & & . & . & . & . & . & 33 & 30 & 32 \\
\hline Slovakia & . & . & . & 62 & 55 & 53 & 55 & 57 & 59 & 61 & 57 & 56 & 58 & 47 & 34 & 32 & 32 & 33 & 33 & 32 \\
\hline Slovenia & . & . & . & . & . & . & . & 64 & 60 & 60 & 51 & 47 & 74 & 70 & 61 & 60 & 59 & 56 & 58 & 57 \\
\hline Spain & 51 & 47 & 44 & 42 & 40 & 40 & 37 & 36 & 35 & 32 & 34 & 36 & 35 & 35 & 35 & 35 & 35 & 35 & 34 & 34 \\
\hline Sweden & 61 & 56 & 58 & 59 & 59 & 59 & 58 & 50 & 47 & 46 & 44 & 43 & 44 & 44 & 45 & 43 & 43 & 42 & 40 & 39 \\
\hline Switzerland & 39 & 39 & 40 & 38 & 38 & 38 & 38 & 38 & 39 & 42 & 41 & 41 & 41 & 40 & 39 & 33 & 32 & 31 & 31 & 31 \\
\hline United Kingdom & 38 & 39 & 39 & 40 & 41 & 40 & 39 & 38 & 38 & 39 & 39 & 38 & 39 & 39 & 40 & 37 & 39 & 39 & 40 & 42 \\
\hline United States & 35 & 33 & 34 & 33 & 32 & 32 & 32 & 31 & 30 & 27 & 27 & 26 & 26 & 25 & 25 & 25 & 24 & 24 & 24 & 23 \\
\hline
\end{tabular}




\section{References}

Allan, J. P., and Scruggs, L. (2004). Political partisanship and welfare state reform in advanced industrial societies. American Journal of Political Science, 48(3), 496-512.

Been, J., and Van Vliet, O. (2017). Early retirement across Europe. Does non-standard employment increase participation of older workers? Kyklos, 70(2), forthcoming.

Eardley, T., Bradshaw, J., Ditch, J., and Gough, I. (1996). Social assistance in OECD countries: Synthesis report. HMSO Research Report no.47. London: Department of Social Security.

European Commission. (2014). Exchange Rates Database. Brussels.

Ferrarini, T., Nelson, K., Korpi, W., and Palme, J. (2013). Social citizenship rights and social insurance replacement rate validity: pitfalls and possibilities. Journal of European Public Policy, 20(9), 12511266.

Heston, A., Summers, R. and Aten, B. (2012). Penn World Table Version 7.1. Pennsylvania: University of Pennsylvania.

Hicks, A., and Freeman, K. (2009). Pension income replacement: permanent and transitory determinants. Journal of European Public Policy, 16(1), 127-143.

Immervoll, H. (2009). Minimum-income benefits in OECD countries: Policy design, effectiveness and challenges. IZA Discussion Papers no. 4627.

Korpi, W., and Palme, J. (2003). New politics and class politics in the context of austerity and globalization: Welfare state regress in 18 countries, 1975-95. American Political Science review, 97(03), 425-446.

Kuivalainen, S. (2003). How to compare the incomparable: an international comparison of the impact of housing costs on levels of social assistance. European Journal of Social Security, 5(2), 128-149.

Marx, I., and Nelson, K. (2013). Minimum Income Protection in Flux. Basingstoke: Palgrave Macmillan.

Nelson, K. (2013). Social assistance and EU poverty thresholds 1990-2008. Are European welfare systems providing just and fair protection against low income? European Sociological Review, 29(2), 386-401.

Olaskoaga, J., Alaez-Aller, R., and Diaz-De-Basurto-Uraga, P. (2013). Beyond welfare effort in the measuring of welfare states. Journal of Comparative Policy Analysis: Research and Practice, 15(3), 274-287.

Scruggs, L., Detlef, J. and Kuitto, K. 2014. Comparative Welfare Entitlements Dataset 2: Version 201403. University of Connecticut and University of Greifswald.

Van Mechelen, N., Marchal, S., Goedeme, T., Marx, I., and Cantillon, B. (2011). The CSB-Minimum Income Protection Indicators Dataset (CSB-MIPI). CSB Working Paper no. 11/05. 
Van Vliet, O., and Caminada, K. (2012). Unemployment Replacement Rates Dataset among 34 Welfare States 1971-2009: An Update, Extension and Modification of Scruggs' Welfare State Entitlements Data Set. NEUJOBS Special Report no. 2.

Van Vliet, O., and Wang, C. (2015). Social investment and poverty reduction: A comparative analysis across 15 European countries. Journal of Social Policy 44(3): 611-638.

Wang, J., and Van Vliet, O. (2014). Social assistance and minimum income benefits: Benefit levels, replacement rates and policies across 33 countries, 1990-2009. Department of Economics Research Memorandum no. 2014.04. Leiden: Leiden University.

Wang, J., and Van Vliet, O. (2016). Social assistance and minimum income benefits: Benefit levels, replacement rates and policies across 26 OECD countries, 1990-2009. European Journal of Social Security 18(4): 333-555.

Wang J., Van Vliet, O. and Goudswaard, K. (2015). Social assistance benefits and European coordination. Department of Economics Research Memorandum no. 2015.02. Leiden: Leiden University.

Whiteford, P. (1995). The use of replacement rates in international comparisons of benefit systems. International Social Security Review, 48(2), 3-30.

World Bank. (2012). World Development Indicators. Washington. 